\title{
Products of Harmonic Polynomials and Delta Functions
}

\author{
Ricardo Estrada \\ Department of Mathematics, Louisiana State University, Baton Rouge, LA 70803, United States \\ Email: restrada@math.1su.edu
}

\begin{abstract}
We prove that the product $p(\mathbf{x}) \nabla^{2 m} \delta(\mathbf{x})$ of a homogeneous polynomial of degree $j$ in $n$ variables, $p$, and the iterated Laplacian of the Dirac delta function is of the form $p(\nabla) q(\nabla) \delta(\mathbf{x})$ for some polynomial $q$ if and only if $p(\mathbf{x})=|\mathbf{x}|^{2 l} p_{k}(\mathbf{x})$ for some harmonic homogeneous polynomial of some degree $k, j=2 l+k$.

We also show that the product $p(\mathbf{x}) \nabla^{2 m} \delta(\mathbf{x})$, where $p$ is homogeneous of degree $j$, vanishes for all $m>j$ if and only if $p$ is a harmonic polynomial.
\end{abstract}

Keywords: Dirac delta function, harmonic polynomials, products of distributions.

\section{Introduction and Notation}

The aim of this short article is to consider several questions associated to the structure of the product of a polynomial and a distribution concentrated at the origin. Products of this kind are found very frequently in many areas, such as the distributional solution of differential equations [11], [15], [16], and in many of the applications of distribution theory, particularly in Mathematical Physics ${ }^{1}$ [2], [3], [10], [12], [13], [17], [18]. For example, the known formulas for the derivatives of power potentials [5], [6] often need to be multiplied by polynomials in the computations performed in the Physics literature [10], [14].

If one considers the product

$$
p(\mathbf{x}) \nabla^{2 m} \delta(\mathbf{x}),
$$

where $p$ is a polynomial in $n$ variables, it is sometimes true that (1.1) equals $p(\nabla) q(\nabla) \delta(\mathbf{x})$ for some polynomial $q$, where $\nabla=\left(\partial / \partial x_{i}\right)_{i=1}^{n}$ is the gradient. Consider for instance the formulas

$$
\begin{gathered}
x_{i} \nabla^{2 m} \delta(\mathbf{x})=-2 m \nabla_{i} \nabla^{2 m-2} \delta(\mathbf{x}), \\
\left(x_{i}^{2}-x_{j}^{2}\right) \nabla^{2 m} \delta(\mathbf{x})=2 m(2 m-2)\left(\nabla_{i}^{2}-\nabla_{j}^{2}\right) \nabla^{2 m-4} \delta(\mathbf{x}),
\end{gathered}
$$

or

$$
x_{i} r^{2} \nabla^{2 m} \delta(\mathbf{x})=-2 m(2 m-2)(2 m-2+n) \nabla_{i} \nabla^{2 m-4} \delta(\mathbf{x}) .
$$

where, as usual, we denote $r=|\mathbf{x}|$. We see that in each case the right hand side is of the form $p(\nabla) q(\nabla) \delta(\mathbf{x})$ for some polynomial $q$. However, this is not always the case, as the example

$$
x_{i}^{2} \nabla^{2 m} \delta(\mathbf{x})=2 m \nabla^{2 m-2} \delta(\mathbf{x})+2 m(2 m-2) \nabla_{i}^{2} \nabla^{2 m-4} \delta(\mathbf{x}),
$$

shows. We give the answer to the question of when this happens in the Proposition 3.4. Several interesting formulas and facts involving the products of polynomials and distributions concentrated at a point are also given in our analysis.

Let us now say a couple of things about the terminology employed. We shall use the notation

$$
C=\frac{2 \pi^{n / 2}}{\Gamma(n / 2)},
$$

\footnotetext{
${ }^{1}$ Unfortunately this is an area where the handling of distributional expressions is required, but many times leaves a lot to be desired. One of our aims is to show how these computations can be done in a solid mathematical way.
} 
for the surface area of the unit sphere $\mathbb{S}$ of $\mathbb{R}^{n}$. We shall employ the basic results for distributions found in the standard texts [7], [11], [15], [16]. Notice that distributional derivatives are denoted with an overbar ${ }^{2}$, namely, $\bar{\partial} / \partial x_{i}, \bar{\nabla}$, or $\bar{\Delta}$.

The space of homogeneous polynomials of degree $k$ in $n$ variables will be denoted as $\mathcal{P}_{k}$. Its subspace $\mathcal{H}_{k}$ is formed by the harmonic homogeneous polynomials of degree $k$. See [1], [19] for the properties of $\mathcal{H}_{k}$. We shall employ the following natural inner product defined in the space $\mathcal{P}_{k}$ of homogeneous polynomials of degree $k$ in $n$ variables,

$$
\{p, q\}=\sum_{|\alpha|=k} \alpha ! a_{\alpha} \bar{b}_{\alpha}
$$

if $p(\mathbf{x})=\sum_{|\alpha|=k} a_{\alpha} \mathbf{x}^{\alpha}$ and $q(\mathbf{x})=\sum_{|\alpha|=k} b_{\alpha} \mathbf{x}^{\alpha}$. Notice that $\{p, q\}$ actually equals the following constant function,

$$
\{p, q\}=p(\nabla) \overline{q(\mathbf{x})} .
$$

\section{Basic Formulas}

The formula for the product of a smooth function $p$ and a derivative of the Dirac delta function in one variable is very simple, namely [16]

$$
p(x) \delta^{(m)}(x)=\sum_{j=0}^{m}\left(\begin{array}{c}
m \\
j
\end{array}\right)(-1)^{j} p^{(j)}(0) \delta^{(m-j)}(x) .
$$

One can prove such identities by evaluation at a test function:

$$
\begin{gathered}
\left\langle p(x) \delta^{(m)}(x), \phi(x)\right\rangle=\left\langle\delta^{(m)}(x), p(x) \phi(x)\right\rangle=\left.(-1)^{m} \frac{d^{m}}{d x^{m}}(p(x) \phi(x))\right|_{x=0} \\
=(-1)^{m} \sum_{j=0}^{m}\left(\begin{array}{c}
m \\
j
\end{array}\right) p^{(j)}(0) \phi^{(m-j)}(0)=\left\langle\sum_{j=0}^{m}\left(\begin{array}{c}
m \\
j
\end{array}\right)(-1)^{j} p^{(j)}(0) \delta^{(m-j)}(x), \phi(x)\right\rangle,
\end{gathered}
$$

for $\phi \in \mathcal{D}(\mathbb{R})$, but once basic identities are established there is no need to evaluate at test functions anymore, since other identities can be obtained by manipulation of the basic ones.

Formula (2.1) yields, in particular, the well known relations

$$
x^{k} \delta^{(n)}(x)=\left\{\begin{array}{cl}
0, & k>n \\
\frac{n !}{(n-k) !}(-1)^{k} \delta^{(n-k)}(x), & k \leq n .
\end{array}\right.
$$

In several variables there is a simple generalization of $(2.2)$, which we may write as

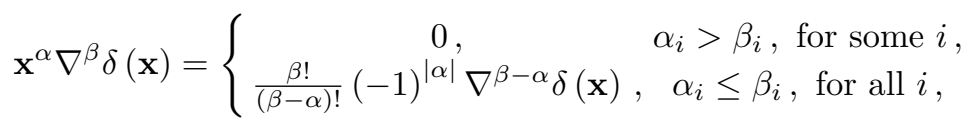

for $\alpha, \beta \in \mathbb{N}^{n}$, since $\mathbf{x}^{\alpha} \nabla^{\beta}=x_{1}^{\alpha_{1}} \nabla_{1}^{\beta_{1}} \cdots x_{n}^{\alpha_{n}} \nabla_{n}^{\beta_{n}}$. We can actually rephrase this formula as follows.

Proposition 2.1 Let $p$ and $q$ be two polynomials in $n$ variables. Then

$$
p(\mathbf{x}) q(\nabla) \delta(\mathbf{x})=\widetilde{q}(\nabla) \delta(\mathbf{x}),
$$

where

$$
\widetilde{q}(\mathbf{x})=p(-\nabla) q(\mathbf{x}) .
$$

\footnotetext{
${ }^{2}$ The overbar notation was first introduced by the late Professor Farassat [8].
} 
Proof. Indeed, (2.3) says that (2.4) holds when $p(\mathbf{x})=\mathbf{x}^{\alpha}$ and $q(\mathbf{x})=\mathbf{x}^{\beta}$. The result is thus obtained because all polynomials are linear combinations of monomials.

Notice that when $p$ is homogeneous of degree $k, p \in \mathcal{P}_{k}$, then $\widetilde{q}(\mathbf{x})=(-1)^{k} p(\nabla) q(\mathbf{x})$.

Let us consider several illustrations of (2.4). We have,

$$
\begin{gathered}
x_{i} \nabla^{2 m} \delta(\mathbf{x})=-2 m \nabla_{i} \nabla^{2 m-2} \delta(\mathbf{x}), \\
x_{i} x_{j} \nabla^{2 m} \delta(\mathbf{x})=2 m(2 m-2) \nabla_{i} \nabla_{j} \nabla^{2 m-4} \delta(\mathbf{x}), \quad i \neq j, \\
x_{1}^{2} \nabla^{2 m} \delta(\mathbf{x})=2 m \nabla^{2 m-2} \delta(\mathbf{x})+2 m(2 m-2) \nabla_{1}^{2} \nabla^{2 m-4} \delta(\mathbf{x}),
\end{gathered}
$$

and

$$
r^{2} \nabla^{2 m} \delta(\mathbf{x})=2 m(2 m-2+n) \nabla^{2 m-2} \delta(\mathbf{x}) .
$$

Notice that for $p$ equal to $x_{i}, x_{i} x_{j}, i \neq j$, or for $r^{2}$, for $m \geq 2$, we have that $\widetilde{q}(\mathbf{x})=p(-\nabla) r^{2 m}$ is actually a multiple of $p(\mathbf{x})$, but for $x_{1}^{2}$ this is not the case. Similarly,

$$
x_{j} r^{2} \nabla^{2 m} \delta(\mathbf{x})=-2 m(2 m-2)(2 m-2+n) \nabla_{j} \nabla^{2 m-4} \delta(\mathbf{x}),
$$

so that $\widetilde{q}(\mathbf{x})$ is a multiple of $p(\mathbf{x})=x_{j} r^{2}$, for $m \geq 4$, while $\widetilde{q}(\mathbf{x})$ is not a multiple of $p(\mathbf{x})=x_{1}^{3}$ since

$$
x_{1}^{3} \nabla^{2 m} \delta(\mathbf{x})=-6 m(2 m-2) \nabla_{1} \nabla^{2 m-4} \delta(\mathbf{x})-2 m(2 m-2) \nabla_{1}^{3} \nabla^{2 m-6} \delta(\mathbf{x}) .
$$

We shall explain this situation in the next section.

\section{Special Products}

We now compute several special products of polynomials and derivatives of the delta function in $\mathbb{R}^{n}$, namely, products of the type $p(\mathbf{x}) \nabla^{2 m} \delta(\mathbf{x})$. Let us start with the case when $p(\mathbf{x})=|\mathbf{x}|^{2 l}$.

Proposition 3.1 If $l>m$ then $r^{2 l} \nabla^{2 m} \delta(\mathbf{x})=0$, while if $l \leq m$,

$$
r^{2 l} \nabla^{2 m} \delta(\mathbf{x})=\frac{2^{2 l} m ! \Gamma(m+n / 2)}{(m-l) ! \Gamma(m-l+n / 2)} \nabla^{2 m-2 l} \delta(\mathbf{x}) .
$$

Proof. Indeed, the Proposition 2.1 gives $r^{2 l} \nabla^{2 m} \delta(\mathbf{x})=\widetilde{q}(\nabla) \delta(\mathbf{x})$, where $\widetilde{q}$ is given by $\widetilde{q}(\mathbf{x})=\nabla^{2 l} r^{2 m}$, which is 0 if $l>m$ and gives (3.1) if $l \leq m$, since

$$
\nabla^{2 l} r^{2 m}=(2 m) \cdots(2 m-2 l+2)(2 m+n-2) \cdots(2 m-2 l+n) r^{2 m-2 l},
$$

as follows by iteration of $\nabla^{2} r^{2 m}=2 m(2 m-2+n) r^{2 m-2}$.

Next we would like to consider the product $p(\mathbf{x}) \nabla^{2 m} \delta(\mathbf{x})$ when $p$ is a harmonic homogeneous polynomial of degree $k$. We need several preliminary results. First, we recall the following formula [4, Eqn. (3.8)]: if $p \in \mathcal{H}_{k}$ and $q \in \mathcal{P}_{k+2 m}$ then

$$
\left\{r^{2 m} p(\mathbf{x}), q(\mathbf{x})\right\}=\frac{W_{n, k, m}}{C} \int_{\mathbb{S}} p(\omega) \bar{q}(\omega) \mathrm{d} \sigma(\omega)
$$

where $W_{n .0,0}=1$ while if $k+m>0$,

$$
W_{n . k, m}=2^{m} m ! n(n+2) \cdots(n+2 k+2 m-2) .
$$

We can now give a proof of the ensuing identity.

Proposition 3.2 Let $p \in \mathcal{H}_{k}$. If $m<k$ then $p(\nabla) r^{2 m}=0$, while if $m \geq k$,

$$
W_{n, k, m-k} p(\nabla) r^{2 m}=W_{n, 0, m} r^{2 m-2 k} p(\mathbf{x}) .
$$


Proof. Notice that $p(\nabla) r^{2 m}$ is a homogeneous polynomial of degree $2 m-k$. If $q \in \mathcal{P}_{2 m-k}$ is an arbitrary homogeneous polynomial of degree $2 m-k$ we have ${ }^{3}$

$$
\left\{p(\nabla) r^{2 m}, q(\mathbf{x})\right\}=\left\{r^{2 m}, p(\mathbf{x}) q(\mathbf{x})\right\}=\frac{W_{n, 0, m}}{C} \int_{\mathbb{S}} p(\omega) \bar{q}(\omega) \mathrm{d} \sigma(\omega) .
$$

This, we notice, vanishes if $m<k$. On the other hand, if $m \geq k$,

$$
\left\{r^{2 m-2 k} p(\mathbf{x}), q(\mathbf{x})\right\}=\frac{W_{n, k, m-k}}{C} \int_{\mathbb{S}} p(\omega) \bar{q}(\omega) \mathrm{d} \sigma(\omega) .
$$

Therefore $W_{n, k, m-k}\left\{p(\nabla) r^{2 m}, q\right\}=W_{n, 0, m}\left\{r^{2 m-2 k} p, q\right\}$ for all $q \in \mathcal{P}_{2 m-k}$, so that (3.5) follows.

If we now combine the previous proposition and the Proposition 2.1, we obtain the next identity ${ }^{4}$.

Proposition 3.3 Let $p \in \mathcal{H}_{k}$. If $m<k$ then $p(\mathbf{x}) \nabla^{2 m} \delta(\mathbf{x})=0$, while if $m \geq k$ then

$$
p(\mathbf{x}) \nabla^{2 m} \delta(\mathbf{x})=\frac{(-1)^{k} 2^{k} m !}{(m-k) !} p(\nabla) \nabla^{2 m-2 k} \delta(\mathbf{x}) .
$$

Proof. Indeed, $p(\mathbf{x}) \nabla^{2 m} \delta(\mathbf{x})=\widetilde{q}(\nabla) \delta(\mathbf{x})$, where $\widetilde{q}$ is given by $\widetilde{q}(\mathbf{x})=(-1)^{k} p(\nabla) r^{2 m}$, which vanishes if $m<k$ and which (3.5) gives as

$$
\widetilde{q}(\mathbf{x})=(-1)^{k} \frac{W_{n, 0, m}}{W_{n, k, m-k}} p(\mathbf{x}) r^{2 m-2 k}
$$

if $m \geq k$; formula (3.8) follows since $W_{n, 0, m} / W_{n, k, m-k}=2^{k} m ! /(m-k)$ !.

We may combine formulas (3.1) and (3.8) to obtain the identity ${ }^{5}$

$$
p_{k}(\mathbf{x}) r^{2 l} \nabla^{2 m} \delta(\mathbf{x})=\frac{(-1)^{k} 2^{2 l+k} m ! \Gamma(m+n / 2)}{(m-l-k) ! \Gamma(m-l+n / 2)} p_{k}(\nabla) \nabla^{2 m-2 l-2 k} \delta(\mathbf{x})
$$

for $p_{k} \in \mathcal{H}_{k}, m \geq k+l$. Notice that this shows that if $p=p_{k} r^{2 l}$ then $\widetilde{q}=p r^{2 m-4 l-2 k}$ is a multiple of $p$ for $m$ large enough.

It is now possible to give a formula for the product $p(\mathbf{x}) \nabla^{2 m} \delta(\mathbf{x})$ for an arbitrary homogeneous polynomial of degree $k, p \in \mathcal{P}_{k}$. Indeed [1], [9] any $p \in \mathcal{P}_{k}$ admits a unique decomposition of the type

$$
p=\sum_{j=0}^{\llbracket k / 2 \rrbracket} r^{2 j} p_{k-2 j}
$$

for some harmonic homogeneous polynomials $p_{k-2 j} \in \mathcal{H}_{k-2 j}$. We thus obtain

$$
p(\mathbf{x}) \nabla^{2 m} \delta(\mathbf{x})=m ! \Gamma(m+n / 2)(-1)^{k} 2^{k} \sum_{j=0}^{\llbracket k / 2 \rrbracket} \frac{p_{k-2 j}(\nabla) \nabla^{2 m+2 j-2 k} \delta(\mathbf{x})}{(m+j-k) ! \Gamma(m-j+n / 2)} .
$$

for $m \geq k-\llbracket k / 2 \rrbracket$. Therefore, we obtain the ensuing result.

Proposition 3.4 If $p \in \mathcal{P}_{k}, p(\mathbf{x}) \nabla^{2 m} \delta(\mathbf{x})=\widetilde{q}(\nabla) \delta(\mathbf{x})$, and $\widetilde{q}$ is a polynomial multiple of $p$ for $m \geq m_{0}$, then

$$
p=r^{2 j} p_{k-2 j}
$$

for some $j \in \mathbb{N}$ and some $p_{k-2 j} \in \mathcal{H}_{k-2 j}$.

\footnotetext{
${ }^{3}$ Since [1], [9] $\left\{\nabla_{i} p, q\right\}=\left\{p, x_{i} q\right\}$.

${ }^{4}$ Another proof can be given by taking Fourier transforms in (3.5). Actually alternative derivations of our formulas can be obtained by using Fourier transforms and the Funk-Hecke formula [19, (A.7.3)].

${ }^{5}$ The product vanishes if $m<k+l$.
} 


\section{A Characterization of Harmonic Polynomials}

The product of a homogeneous polynomial of degree $k, p(\mathbf{x})$, and a distribution concentrated at a point, $q(\nabla) \delta(\mathbf{x})$, where $q$ has degree $m$, vanishes if $k>m$. However, unlike the one dimensional case, in several variables the product could vanish when $k \leq m$, even if the degree of $p$ is much smaller than that of $q$. Consider for example the product $r^{2} q(\nabla) \delta(\mathbf{x})$, that vanishes if $q \in \mathcal{H}_{m}$, no matter how large $m$ is. A particularly interesting case is the product $p(\mathbf{x}) \nabla^{2 m} \delta(\mathbf{x})$ when $p \in \mathcal{H}_{k}$, that vanishes whenever $m<k$; actually this fact characterizes the harmonic polynomials among the homogeneous polynomials of degree $k$.

Proposition 4.1 Let $p \in \mathcal{P}_{k}$. Then $p \in \mathcal{H}_{k}$ if and only if

$$
p(\mathbf{x}) \nabla^{2 m} \delta(\mathbf{x})=0, \quad m<k .
$$

Proof. Indeed, suppose $p \in \mathcal{P}_{k} \backslash \mathcal{H}_{k}$. Then when we decompose $p$ as in (3.11), there must be at least one $j>0$ such that $p_{k-2 j} \neq 0$. Formula (3.12) then shows that $p(\mathbf{x}) \nabla^{2 k-2 j} \delta(\mathbf{x}) \neq 0$.

\section{References}

1. Axler, S., Bourdon, P., and Ramey, W., Harmonic Function Theory, second edition, Springer, New York, 2001.

2. Blinder, S.M., Delta functions in spherical coordinates and how to avoid losing them: Fields of point charges and dipoles, American Journal of Physics 71 (2003), 816-818.

3. Bowen, J. M., Delta function terms arising from classical point-source fields, American Journal of Physics 62 (1994), 511-515.

4. Estrada, R., Regularization and derivatives of multipole potentials, Journal of Mathematical Analysis and Applications 446 (2016), 770-785.

5. Estrada, R. and Kanwal, R. P., Regularization and distributional derivatives of $\left(x_{1}^{2}+\cdots x_{p}^{2}\right)^{n / 2}$ in $\mathcal{D}^{\prime}\left(\mathbb{R}^{p}\right)$, Proceedings of the Royal Society of London A 401 (1985), 281-297.

6. Estrada, R. and Kanwal, R. P., Regularization, pseudofunction, and Hadamard finite part, Journal of Mathematical Analysis and Applications 141 (1989), 195-207.

7. Estrada, R. and Kanwal, R.P., A distributional approach to Asymptotics. Theory and Applications, second edition, Birkhäuser, Boston, 2002.

8. Farassat, F., Introduction to generalized functions with applications in aerodynamics and aeroacoustics, NASA Technical Paper 3248 (Hampton, VA: NASA Langley Research Center) (1996); http://ntrs.nasa.gov.

9. Folland, G.B., Introduction to Partial Differential Equations, Princeton University Press, Princeton, 1976.

10. Frahm, C.P., Some novel delta-function identities, American Journal of Physics 51 (1983), 826-29.

11. Gel'fand, I. M. and Shilov, G. E., Generalized functions, Vol. 1. Properties and operations, Academic Press, New York-London, 1964.

12. Gsponer, A., Distributions in spherical coordinates with applications to classical electrodynamics, European Journal of Physics 28 (2007), 267-75; European Journal of Physics 28 (2007), 1241 (corrigendum).

13. Hnizdo, V., Generalized second-order partial derivatives of 1/r, European Journal of Physics 32 (2011), $287-297$.

14. Jackson, J. D., Classical Electrodynamics, Third Edition, Wiley, New York, 1998.

15. Jones, D. S., Generalised Functions, Cambridge Univ. Press, London-New York, 1982.

16. Kanwal, R.P., Generalized Functions: Theory and Technique, Third Edition, Birkhäuser, Boston, 2004.

17. Namias, V., Singularities in the fields of electric and magnetic multipoles and related identities involving the Dirac delta function, International Journal of Education in Science and Technology 18 (1987), 767-781.

18. Parker, E., An apparent paradox concerning the field of an ideal dipole. Preprint, arXiv:1604.01121v1.

19. Rubin, B., Introduction to Radon transforms (with elements of Fractional calculus and Harmonic Analysis), Cambridge University Press, Cambridge, 2015. 